\title{
DISTRIBUIÇÃO E INCERTEZA DA PRECIPITAÇÃO PLUVIOMÉTRICA E DO POTENCIAL EROSIVO DAS CHUVAS PARA O ESTADO DA BAHIA, BRASIL
}

\author{
1 - Engenheira Agrônoma, UESC/Ilhéus, BA, soares.yasmine@gmail.com \\ 2 - Engenheiro Agrônomo, UESC/Ilhéus, BA, matheus.lopes@outlook.com \\ 3 - Engenheira Agrônoma, Professor Adjunto, UFES/Alegre, ES, sasilva@pq.cnpq.br \\ 4 - Engenheira Agrônoma, UENF/Campos dos Goytacazes, RJ, danielle.inacio@hotmail.com \\ 5 - Engenheiro Agrícola, Professor Titular, UFES/Alegre, ES, limajss@yahoo.com.br
}

Yasmine Soares Cardoso ${ }^{1}$, Matheus Reis Lopes², Samuel de Assis Silva ${ }^{3}$, Danielle Inacio Alves ${ }^{4}$ \& Julião Soares de Souza Lima

\section{Palavras-chave: conservação do solo erosividade geoestatística lógica fuzzy variabilidade espacial}

\section{RESUMO}

As alterações climáticas e as suas consequências vêm sendo uma das grandes preocupações da humanidade e, neste tocante, a precipitação pluviométrica tem atenção espacial nos estudos ambientais. Com este trabalho, objetivou-se utilizar uma ponderação por meio da modelagem fuzzy e métodos geoestatísticos para estudar a propagação de incertezas da precipitação pluviométrica e do potencial erosivo das chuvas para o estado da Bahia. Foram utilizados os valores de precipitação pluvial levantados para 71 postos pluviométricos localizados na área do estado, avaliando-se a média anual das séries históricas usadas. Para cada posto pluviométrico, foram calculados os valores de erosividade, através da relação entre a precipitação média mensal e a precipitação média acumulada anual. Os dados foram submetidos primeiramente à análise estatística clássica, seguida da geoestatística para modelar a variabilidade espacial dos fenômenos estudados. Foi realizada uma codificação para modelagem através de krigagem por indicação e também classificação segundo modelos fuzzy, utilizando para a precipitação modelos exponenciais e para a erosividade modelos lineares. A lógica fuzzy se mostrou mais eficiente nos estudos ambientais quando comparada à krigagem por indicação. Os valores resultantes das análises de erosividade mostrou que, na maior parte do estado, as chuvas possuem elevado potencial para causar erosão, com um limite acima do aceitável.

\section{Keywords:}

soil conservation

erosivity

geostatistics

fuzzy logic

spatial variability

\section{DISTRIBUTION AND UNCERTAINTY OF RAINFALL AND THE EROSIVITY FOR} BAHIA STATE, BRAZIL

\section{ABSTRACT}

The climate change and its consequences have been a major concern of humankind and, in this regard, rainfall has a spatial attention in environmental studies. This study aimed to use a weighting by fuzzy modeling and geostatistical methods to study the propagation of rainfall and erosive potential uncertainties for the state of Bahia. We used the rainfall values raised to 71 rain gauges located within the state, evaluating the annual average of used historical series. For each rain gauges were calculated the rainfall erosivity values, through the relation between the monthly average rainfall and the accumulated annual average rainfall. The data were firstly submitted to the classical statistical analysis, followed by geostatistics to model the spatial variability of the studied phenomena. The data were further coded for modeling by indicator kriging also classified according to fuzzy models using for exponential models precipitation and linear erosivity models. The fuzzy logic is more efficient in environmental studies when compared to indicator kriging. The resulting values of erosivity analysis showed that in most part of the state, the rains have high potential to cause erosion, with a limit above the acceptable. 


\section{INTRODUÇÃO}

A chuva é um fator preponderante no planejamento agrícola e ambiental. Tão importante quanto o volume precipitado, a intensidade e capacidade de desencadear processos erosivos devem ser consideradas antes e durante a implantação de qualquer atividade, seja ela em ambientes urbanos ou rurais (SOUZA \& AZEVEDO, 2012).

Conhecer a energia cinética associada às gotas da chuva pode possibilitar um correto planejamento de atividades conservacionistas e indicar a aptidão de áreas para a produção agropecuária (CARVALHO \& ASSAD, 2005). Isso se deve ao fato da chuva ser o agente principal em processos de escoamento superficial o que, associado aos solos com baixa resistência à desagregação e às práticas agronômicas inadequadas ou ausência de cobertura vegetal (rugosidade), pode conduzir a processos de degradação irreversíveis ou de difícil reversibilidade.

Devido ao seu caráter aleatório e distribuição irregular tanto em escala temporal quanto espacial, a chuva tem grande potencial para elevar os riscos na programação das atividades do setor agrícola (BRITTO et al., 2008). A obtenção da correta distribuição espacial da precipitação é relevante no planejamento agrícola, no que diz respeito à instalação de culturas e sua influência na agricultura (SILVA \& LIMA, 2011).

No estudo e mapeamento do comportamento espacial da precipitação, diversas são as ferramentas e métodos disponíveis. Aplicações de modelos matemáticos e de modelos estatísticos têm permitido definir satisfatoriamente $o$ comportamento dos fenômenos que ocorrem na natureza, em particular na agricultura. Entretanto, uma vez que cada ambiente e evento são únicos nas escalas temporal e espacial e, consequentemente, os mesmos variam de maneiras diferentes, ainda não se pode estabelecer um método tido como ideal para todas as situações (SILVA et al., 2010a).

COSTA et al. (2012) utilizaram o método Topoto-Raster para espacializar e avaliar a sazonalidade da precipitação pluviométrica no estado de Goiás e Distrito Federal, obtendo resultados satisfatórios na descrição de analogias no regime de chuvas ao longo dos anos, nos territórios avaliados. Da mesma forma, MARCUZZO et al. (2011a), avaliando diferentes métodos de interpolação para o mapeamento de chuvas no estado do Mato Grosso, concluíram que, para as condições de estudo, os melhores resultados foram obtidos com o método Topo-to-Raster.

SILVA \& LIMA (2011), utilizando métodos geoestatísticos uni e multivariados, espacializaram a precipitação pluviométrica no estado do Espírito Santo e obtiveram considerável acurácia nos resultados. Com base no método utilizado e nos resultados obtidos, esses autores propuseram uma metodologia com base na análise variográfica para distribuição de postos pluviométricos para melhor mapear a precipitação no estado.

LIMA et al. (2016) utilizaram métodos geoestatísticos para estudar a variabilidade espacial dos percentis 75 da precipitação pluvial mensal para o estado do Espírito Santo. Os autores descreveram, a partir da metodologia de estudo, a sazonalidade da precipitação pluviométrica, indicando a existência de diferentes microclimas no estado e sua possível influência sobre práticas agronômicas.

Apesar das diferentes ferramentas matemáticas que têm sido utilizados para a espacialização da precipitação e de seu potencial erosivo, são escassos os estudos que utilizam métodos probabilísticos e/ou avaliam os referidos fenômenos a partir de modelos de lógica difusa. A krigagem indicativa permite a definição de áreas e ou regiões com maior ou menor probabilidade que um determinado evento ocorra. Entretanto, por partir de um sistema booleano que considera valores críticos como critério de corte para estabelecimento de uma codificação binária, ao se trabalhar com dados incertos, uma informação deixa de ser representada, dada a dicotomia da modelagem (SILVA et al., 2011). O uso desta teoria pode se tornar inviável em tratamentos de informações imprecisas.

Para a modelagem de sistemas com elevada incerteza, a utilização da teoria dos conjuntos nebulosos, também conhecida como teoria dos conjuntos fuzzy, vem crescendo nos últimos anos em estudos agrícolas e ambientais. Essa metodologia é muito eficiente em fenômenos onde as fronteiras entre classes não são claramente 
definidas ou rígidas (CHIANG \& HSU, 2002), onde vetores de entrada são combinados por regras e atribuídos a um dos conjuntos de classes de saída especificados (SILVA et al., 2010b).

PETKOVIĆ et al. (2017) utilizaram modelos fuzzy para estudar o índice de concentração de precipitação na Sérvia. Esses autores observaram que o mês de outubro é o mais influente para a previsão e estimativa do índice de concentração de precipitação, podendo ser usado para a simplificação de métodos preditivos para estudos da precipitação no país. NADERIANFAR et al. (2017), utilizando a lógica fuzzy, desenvolveram uma metodologia para encontrar os efeitos cumulativos da precipitação e evapotranspiração nas flutuações de níveis de água subterrânea.

Dada as considerações, com este trabalho objetivou-se utilizar uma ponderação por meio da modelagem fuzzy e métodos geoestatísticos para estudar a propagação de incertezas da precipitação pluviométrica e do potencial erosivo das chuvas para o estado da Bahia.

\section{MATERIAL E MÉTODOS}

O estudo foi realizado para o estado da Bahia (Figura 1). Foram utilizados os dados de precipitação pluvial de 24 horas expressas em altura de lâmina d'água ( $\mathrm{mm}$ ), obtidas a partir de 71 postos pluviométricos da Agência Nacional de Águas
(ANA) distribuídas por todo o território do estado da Bahia. A seleção dos postos pluviométricos utilizados seguiu o critério da consistência e disponibilidade de dados de séries históricas maiores ou iguais a uma normal climatológica (30 anos). Os dados foram obtidos a partir do sistema online da ANA, denominado HidroWeb, onde se encontra disponível gratuitamente todo o banco de dados referente aos postos distribuídos pelo estado da Bahia.

Inicialmente, os dados foram agrupados para determinação do valor da precipitação média mensal para cada posto localizado no estado. $\mathrm{O}$ fator de erosividade da chuva $(\mathrm{R})$ foi determinado através do método proposto por Bertoni \& Lombardi Neto (1999), estimado pelo uso da equação 1:

$E I=67,355\left(\frac{r^{2}}{P}\right)^{0,85}$

em que,

$E I=$ média mensal do índice de erosão (MJ.mm.ha${ }^{1} \cdot \mathrm{h}^{-1} \cdot$ ano $\left.^{-1}\right)$

$r=$ precipitação média mensal $(\mathrm{mm})$; e

$P=$ precipitação média acumulada anual $(\mathrm{mm})$.

Para o objetivo desse trabalho, foi considerada apenas a erosividade média anual para cada posto pluviométrico, selecionado conforme os critérios

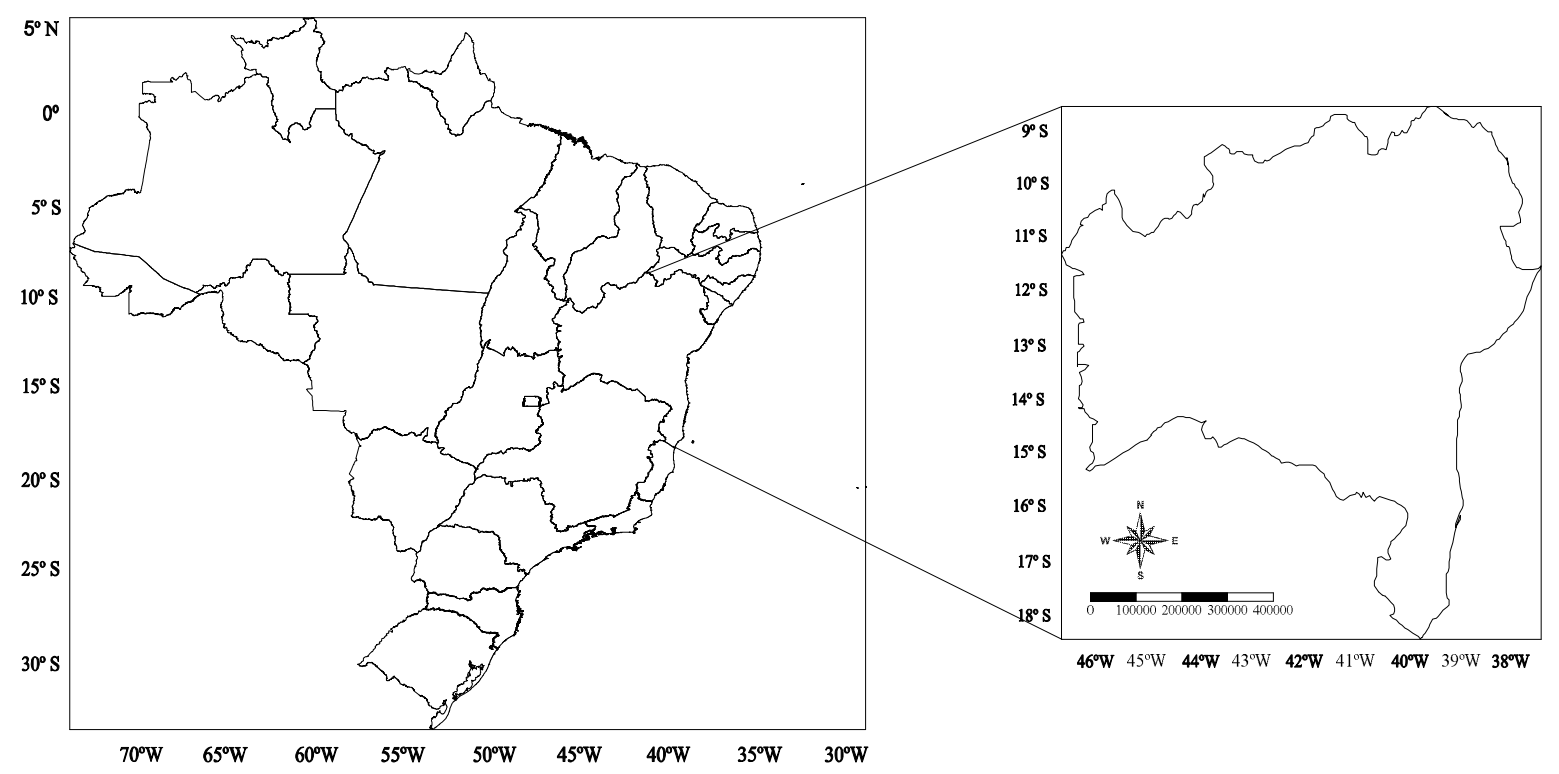

Figura 1. Localização do estado da Bahia no mapa político do Brasil. 
estabelecidos e apresentados anteriormente.

Os dados de precipitação média mensal e de erosividade foram submetidos a uma análise de tendência, avaliando a dispersão dos mesmos em função das direções, conforme SILVA et al. (2009). Após essa determinação, os dados foram submetidos a uma análise estatística descritiva e exploratória, e testada a sua normalidade pelo teste Shapiro-Wilk ao nível de $5 \%$ de probabilidade.

Para aplicação da krigagem indicativa, realizou-se uma transformação não linear sobre o conjunto de dados amostrais para a precipitação e erosividade, denominada codificação por indicação. Essa codificação por indicação é o primeiro passo para realização da krigagem indicativa e consiste em transformar os dados originais em indicadores, isto é, transformar os valores que estão acima de um determinado nível de corte em um (1) e os que estão abaixo, em zero (0).

Neste trabalho, utilizou-se o valor de precipitação equivalente a $1000 \mathrm{~mm} \mathrm{ano}{ }^{-1}$ como valor de corte. Este valor limite foi estabelecido por ser o valor médio de exigência pluviométrica das principais culturas agrícolas cultivadas no estado da Bahia, conforme GRAMACHO et al. (1992) e MENKE et al. (2009). Para a erosividade, utilizouse o valor de 500 MJ.mm.ha $a^{-1} \cdot h^{-1} \cdot$ ano $^{-1}$, o qual foi estabelecido como limite de tolerância conforme RUFINO (1986) e SILVA (2004).

Os dados reais e os valores codificados foram, na sequência, submetidos a uma análise geoestatística para quantificar a dependência espacial, buscandose o atendimento da hipótese de estacionaridade assumida, por meio de ajuste do variograma estimado pela equação 2 :

$\gamma^{*}(h)=\frac{1}{2 N(h)} \sum_{i=1}^{N(h)}\left[z\left(x_{i}\right)-z\left(x_{i}+h\right)\right]^{2}$

em que,

$y^{*}(h)=$ semivariância para um vetor; e

$N(h)=$ número de pares experimentais de observações da precipitação ou erosividade $\mathrm{z}(x i)$, $z(x i+h)$, separados por um vetor $h$.
$\mathrm{Na}$ avaliação da variabilidade espacial, o vetor $\mathrm{h}$ foi representado pela distância física, em metros, entre os pares experimentais $z\left(x_{i}\right)$.

$\mathrm{O}$ ajuste dos modelos teóricos aos variogramas experimentais foi realizado utilizando o software geoestatísticos GS+. Foram determinados os coeficientes: efeito pepita $\left(\mathrm{C}_{0}-\right.$ corresponde ao valor da interseção no eixo das semivariâncias), patamar $\left(\mathrm{C}_{0}+\mathrm{C}_{1}-\right.$ aproximadamente igual ao valor da variância dos dados), variância estrutural $\left(\mathrm{C}_{1}-\right.$ corresponde à variância estrutural) e alcance ( $\mathrm{a}$ representa a distância a partir da qual os dados são independentes), parâmetros fundamentais para a compreensão da estrutura da dependência espacial. $\mathrm{Na}$ escolha dos modelos, foi utilizado o critério dos mínimos quadrados, optando-se na seleção por modelos com maior valor de $\mathrm{R}^{2}$ (coeficiente de determinação), menor SQR (soma de quadrado dos resíduos) e maior valor do coeficiente de correlação obtido pelo método de validação cruzada.

Após a aplicação da análise geoestatística, os dados foram submetidos a procedimentos de interpolação para mapear a estrutura da dependência espacial da precipitação e da erosividade em todo o território do estado da Bahia. A interpolação foi realizada através do interpolador geoestatístico da krigagem ordinária, que estima valores sem tendência e com desvios mínimos em relação aos valores conhecidos, e também por krigagem indicativa (KI), onde foram efetuadas as estimativas de probabilidade baseadas em valores que se situam abaixo ou acima do nível de corte estabelecido e discutido anteriormente.

Visando definir a propagação de incertezas associadas às representações numéricas da precipitação e da erosividade, os seus valores foram integrados para sua espacialização. Nesta etapa, foi necessário, inicialmente, expressá-la de acordo com a classe ideal para o desenvolvimento da maioria das culturas agrícolas cultivadas no estado da Bahia. Conforme discutido anteriormente, a exigência pluviométrica de tais culturas fica entre 800 e $1200 \mathrm{~mm}$, tendo sido, neste trabalho, esse valor utilizado como referência para a codificação fuzzy. 
A representação da precipitação foi submetida a uma classificação contínua utilizando o mapeamento fuzzy. A função de associação escolhida foi a exponencial, apresentada por SILVA et al. (2009).

- Conjunto de dados de precipitação

MF A $(z)=1 /\left(1+d^{*}(z-p) 2\right) \quad$ se $z<p$

$\operatorname{MFA}(\mathrm{z})=1 \quad$ se $\mathrm{p}<\mathrm{z}<\mathrm{q}$

$\operatorname{MF~A~}(\mathrm{z})=1 /\left(1+\mathrm{d}^{*}(\mathrm{z}-\mathrm{q}) 2\right) \quad$ se $\mathrm{z}>\mathrm{q}$

em que,

$\mathrm{d}=\mathrm{q}-\mathrm{p}$;

p e q - valores dos limites de classe de dois conjuntos fuzzy pertencentes a um conjunto A.

Para integrar o comportamento da erosividade, definiu-se como limite o mesmo valor estabelecido como ponto de corte para aplicação da categorização da KI, ou seja, 500 MJ.mm.ha ${ }^{-1} \cdot h^{-1} \cdot$ ano $^{-1}$.

Para a lógica fuzzy, a função de associação escolhida foi a linear, descrita por BÖNISCH et al. (2004) e utilizada por SILVA et al. (2010a):

- Conjunto de dados com valores crescentes

$\operatorname{MFA}(\mathrm{z})=0 \quad$ se $\mathrm{z}<\mathrm{p}$

$\operatorname{MFA}(\mathrm{z})=(1 / \alpha) / \mathrm{z}-\mathrm{p} \quad$ se $\mathrm{p}<\mathrm{z}<\mathrm{q}$

$\operatorname{MFA}(\mathrm{z})=1 \quad$ se $\mathrm{z}=\mathrm{q}$

em que,

$\alpha=\mathrm{q}-\mathrm{p}$;

p e q - valores dos limites de classe de dois conjuntos fuzzy pertencentes a um conjunto $\mathrm{A}$.

A zona de transição fuzzy é dada pela inclinação das retas para o conjunto A com valores exponenciais e para o conjunto B com valores crescentes, conforme figura 2.

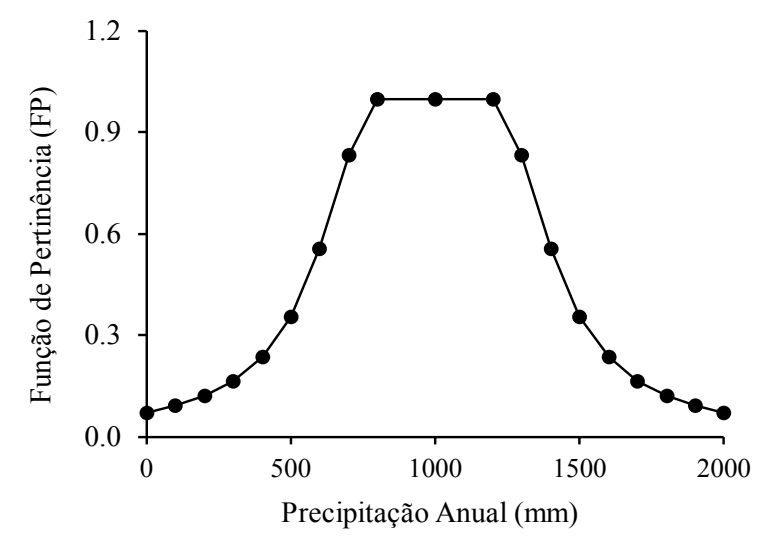

(A)

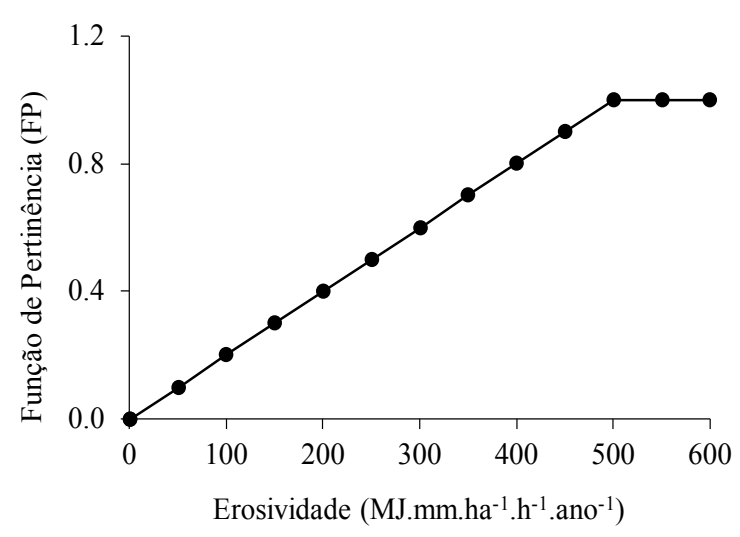

(B)

Figura 2. Conjuntos fuzzy para dados com valores com comportamento exponencial (A) e comportamento crescente linear (B).

Para a precipitação, os valores dos parâmetros $\mathrm{p}, \mathrm{q}$ e $\mathrm{r}$ foram definidos em função dos valores dos limites dos intervalos, relacionados com a classe de precipitação adequada para o desenvolvimento das culturas agrícolas no estado da Bahia. Para a erosividade, os valores dos parâmetros $p, q$ e $r$ foram definidos em função dos limites entre $0 \mathrm{e}$ $500 \mathrm{MJ} \cdot \mathrm{mm} \cdot \mathrm{ha}^{-1} \cdot \mathrm{h}^{-1} \cdot \mathrm{ano}^{-1}$.

\section{RESULTADOS E DISCUSSÃO}

As principais estatísticas da precipitação média anual e da erosividade para o estado da Bahia estão na Figura 3. Os valores de média e mediana para a precipitação apresentaram-se distantes, indicando uma distribuição assimétrica dos dados, com maior concentração à esquerda em relação à curva de distribuição normal, conforme se constata no gráfico de caixas.

O valor médio da erosividade encontra-se muito superior ao limite estabelecido como aceitável (500 MJ.mm.ha $\left.{ }^{-1} \cdot \mathrm{h}^{-1} \cdot \mathrm{ano}^{-1}\right)$. Conforme observado para a precipitação, os valores de tendência central (média e mediana) para a erosividade estão distantes, com valor de média superior à mediana, indicando tendência de concentração dos dados à direita, em relação à curva de distribuição normal.

Para as duas variáveis, os comportamentos das medidas de tendência central são confirmados pelos testes de assimetria e curtose, nos quais o valor de assimetria é positivo, com ligeiro afastamento de zero e o valor de curtose é elevado e negativo, 
indicando pronunciado achatamento da curva de distribuição normal.
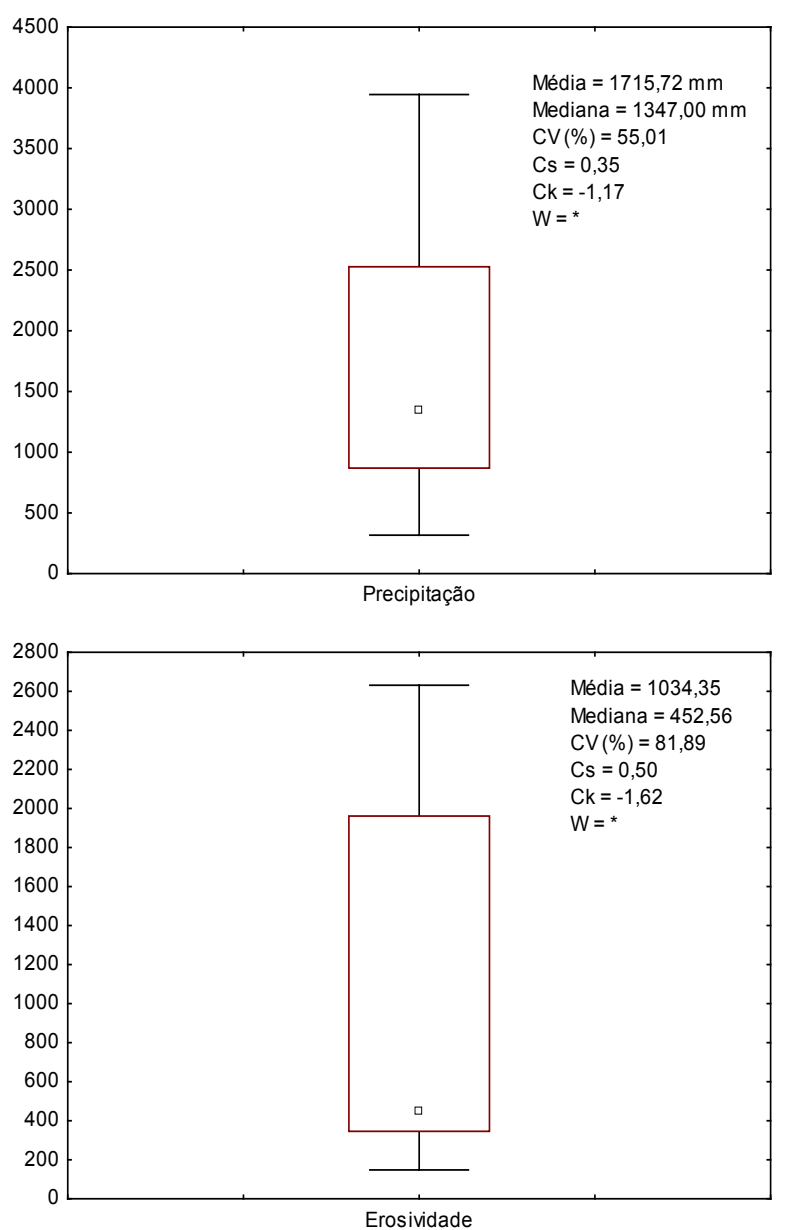

Figura 3. Gráfico de caixa e estatística descritiva para a precipitação média anual e para a erosividade no estado da Bahia.

Ainda na Figura 3, se evidencia também a grande amplitude dos dados, o que já era de se esperar, visto que a variável em estudo apresenta grande variação em função das regiões de amostragens, conforme relatado por SILVA \& LIMA (2011). Dado essa amplitude, observa-se um distanciamento dos dados da normalidade, conforme teste ShapiroWilk $(\mathrm{p} \leq 0,05)$. A normalidade dos dados não é uma exigência na geoestatística, esperando-se apenas que as curvas não apresentem caudas muito alongadas.

Devido à configuração do estado da Bahia e a distribuição de pontos de forma a contemplar todas as regiões, assumiu-se a isotropia dos dados para construção dos variogramas, uma vez que não se observou tendência de distribuição dos dados. Esses resultados se evidenciam nos ajustes, aos variogramas experimentais, de modelos com patamares bem definidos e dentro da maior distância entre os pontos (Tabela 1). LIMA et al. (2010) afirmam que a presença de tendência de distribuição dos dados pode comprometer de forma significativa as interpretações nas análises espaciais, sendo os mapas temáticos uma falsa representação da realidade.

Todas as variáveis apresentaram dependência espacial, com variogramas apresentando patamares definidos. BRAGA et al. (1998) afirmam que o estado da Bahia, devido a sua posição geográfica, é influenciado por sistemas atmosféricos de várias escalas, em diferentes épocas do ano, fazendo com que haja variação significativa de precipitação ao longo do seu território.

O modelo que melhor se ajustou aos dados foi o exponencial, com alcance variando de 62.400 m a 209.700 m. CARVALHO \& ASSAD (2005) ajustaram a essas variáveis o modelo esférico, para o estado de São Paulo, enquanto SILVA et al. (2010a) e MELLO et al. (2012) ajustaram o modelo exponencial para o estado do Espírito Santo. KERRY \& OLIVER (2008) afirmam que modelos exponenciais tendem a apresentar alcances maiores

Tabela 1. Modelos e parâmetros dos variogramas para a precipitação e erosividade para o estado da Bahia.

\begin{tabular}{ccccccccc}
\hline \multirow{2}{*}{ Variáveis } & Modelo & $\mathrm{C}_{0}$ & $\mathrm{C}_{0}+\mathrm{C}$ & $\mathrm{IDE}(\%)$ & $\mathrm{a}(\mathrm{m})$ & $\mathrm{R}^{2}(\%)$ & $\mathrm{RSS}$ & $\mathrm{R}^{2} \mathrm{VC}(\%)$ \\
\cline { 2 - 9 } & Exponencial & 0,4108 & 1,0187 & 59,6 & 94500 & 56,9 & 0,0693 & 25,2 \\
Precipitação & Exponencial & 0,2774 & 1,0707 & 74,1 & 209700 & 64,3 & 0,0753 & 16,2 \\
Precipitação KI & Exponencial & 0,1812 & 1,0686 & 83 & 62400 & 57 & 0,0604 & 20 \\
Erosividade & Exponencial & 0,5165 & 1,037 & 50 & 120300 & 51 & 0,06 & 20,2 \\
Erosividade KI & &
\end{tabular}

Efeito pepita $\left(\mathrm{C}_{0}\right)$, patamar $\left(\mathrm{C}_{0}+\mathrm{C}\right)$, índice de dependência espacial (IDE), alcance (a), coeficiente de determinação ( ${ }^{2}$ ), erro (RSS), coeficiente de Validação Cruzada $\left(\mathrm{R}^{2} \mathrm{VC}\right)$ 
quando comparados a modelos esféricos, uma vez que o patamar é atingido assintoticamente. De acordo com esses autores, isso pode refletir diretamente nos resultados de validação cruzada no processo de interpolação e na qualidade final dos mapas.

O ajuste de modelos teóricos aos experimentais, com patamares bem definidos, indicam 0 atendimento à hipótese geoestatística de estacionaridade de primeira ordem, assegurando a presença de dependência espacial para as variáveis em estudo (KERRY \& OLIVER, 2008). A variabilidade espacial da precipitação tem sido mostrada por diferentes pesquisas científicas, corroborando aos resultados obtidos neste estudo. MARCUZZO et al. (2012) mapearam a precipitação e a sazonalidade das chuvas no estado do Mato Grosso, indicando as anomalias climáticas existentes e os períodos de maiores e menores precipitações. MARCUZZO et al. (2011b), estudando tendências futuras para a precipitação na região amazônica, evidenciaram diminuição nos índices pluviométricos ao longo dos anos.

Estudos sobre a distribuição espaço-temporal da precipitação pluviométrica têm impacto direto sobre a sustentabilidade natural de diferentes ecossistemas. Na agricultura, por exemplo, estudos desta natureza fornecem importantes indicativos sobre a aptidão de áreas para determinadas culturas, bem como permitem explicar variações em índices médios de produtividade (OLIVEIRA \& MARCUZZO, 2016), auxiliando a tomada de decisão para manejos agrícolas.

Analisando os variogramas dos valores reais e os variogramas indicativos, observa-se uma redução do valor de alcance para esses últimos e, consequentemente, aumento da variabilidade espacial. Essa diferença entre os alcances está relacionada à codificação, onde, de acordo com SILVA et al. (2010a), há uma perda na continuidade espacial do fenômeno estudado.

Apesar da redução no valor de alcance para os valores categorizados, os mesmos são elevados e corroboram com os resultados encontrados por outros autores (SILVA et al., 2010a; CARVALHO \& ASSAD, 2005). VIEIRA (2000) afirma que os pontos localizados numa área de raio menor ou igual ao alcance são mais similares, estão dependentes espacialmente entre si e podem ser usados para estimar valores para qualquer ponto entre eles.

Segundo a classificação proposta por ZIMBACK (2001), com exceção da erosividade KI que apresentou forte dependência espacial, as demais variáveis apresentaram dependência moderada. Já os valores de coeficientes de determinação da validação cruzada, para todas as variáveis, indicam que os modelos apresentam confiabilidade no mapeamento das variáveis em estudo.

A distribuição espacial da precipitação pluvial no estado da Bahia é apresentada na Figura 4. É possível observar que a maior proporção do estado apresenta precipitação média anual inferir a 1500 $\mathrm{mm}$ (aproximadamente $76 \%$ do território do estado). Valores superiores a estes são encontrados na porção litorânea do estado e em algumas áreas de maiores altitudes. Esses resultados estão condizentes com aqueles apresentados no Atlas Pluviométrico do Brasil (PINTO et al., 2011), o qual indica que a maior proporção do estado (com exceção de algumas pequenas regiões - em especial o litoral) apresentam precipitação média anual inferior a $1500 \mathrm{~mm}$

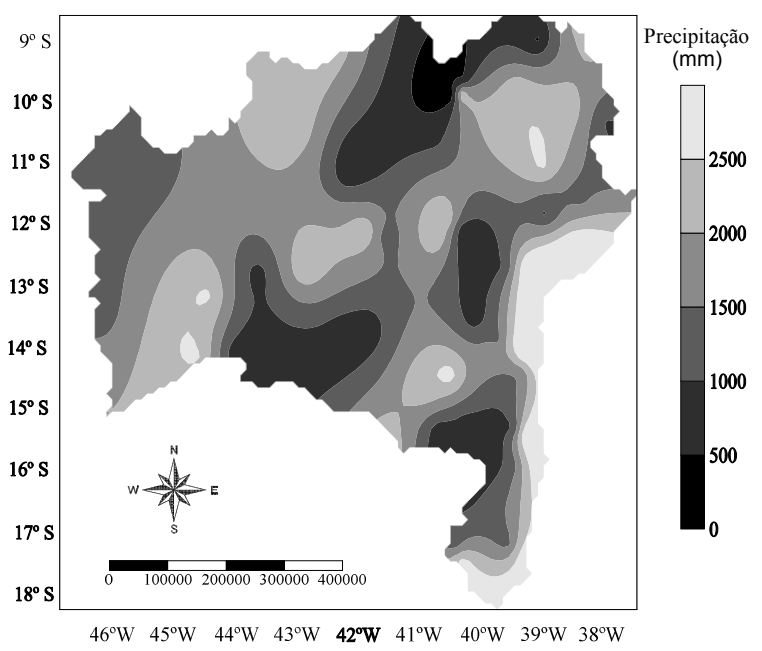

Figura 4. Precipitação pluvial média anual para o estado da Bahia.

Analisando os mapas temáticos (Figura 5), fica evidente que o estado da Bahia possui faixas de precipitação e erosividade diferentes ao longo de seu território. 

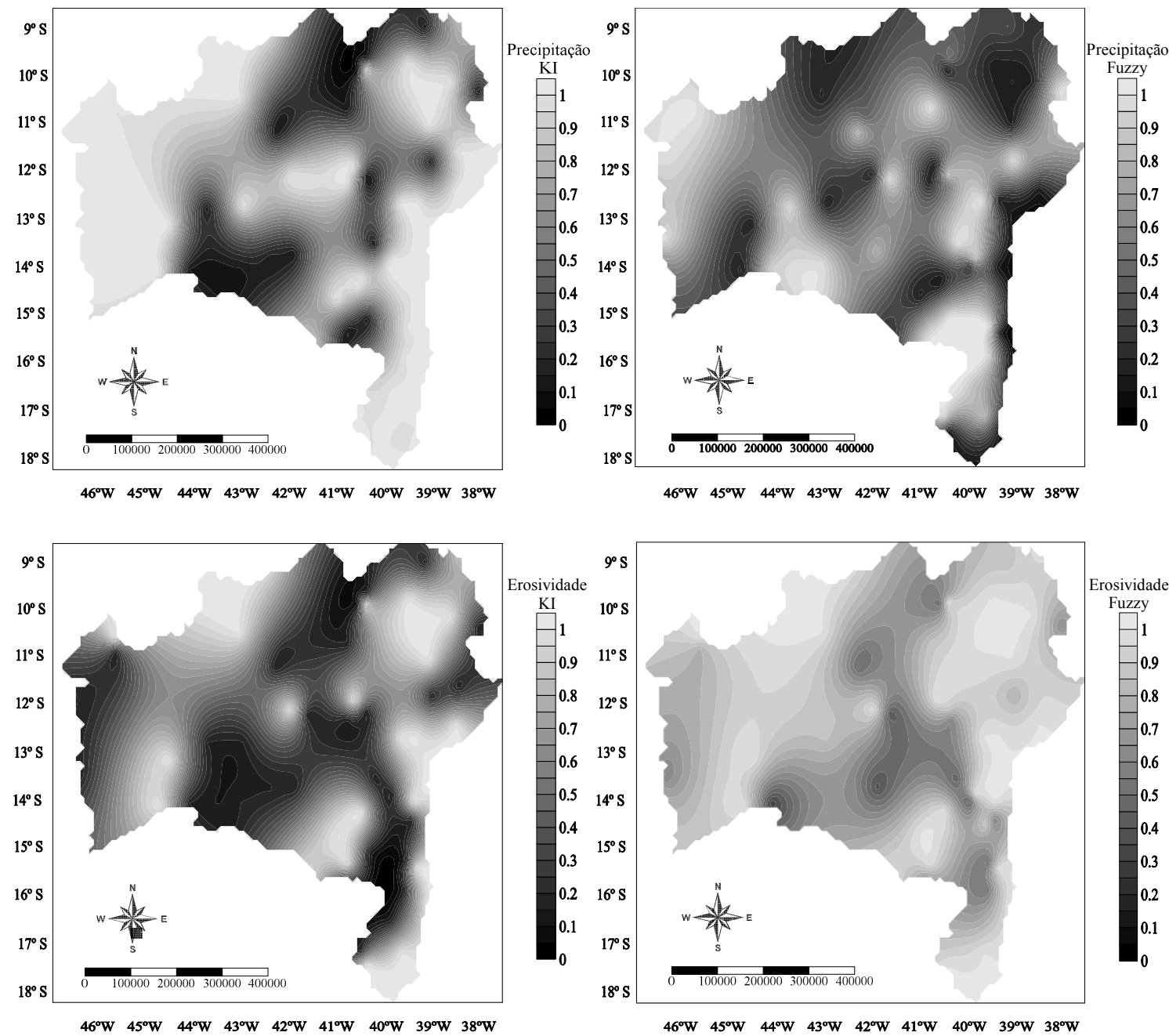

Figura 5. Pertinências da precipitação pluviométrica e erosividade das chuvas estimadas por krigagem indicativa (KI) e integrado por lógica fuzzy.

Os menores índices de precipitação ocorrem em regiões específicas do estado, mas coincidentes com porções do semiárido, corroborando com os resultados de MOURA (2010). Áreas mais próximas ao litoral apresentam os maiores valores de precipitação, os quais podem ser atribuídos, entre outros fatores, à ação direta de massas de ar vindas do oceano, as quais controlam a precipitação nessa região do estado. De acordo com MOLION \& BERNARDO (2000), o Nordeste brasileiro está sobre influencia direta da massa de ar tropical atlântica, a qual atua de forma independente durante o verão, e que no inverno encontra a massa polar atlântica (única massa de ar frio atuante no Brasil), responsável por frequentes chuvas frontais do litoral nordestino nesta época do ano.

Analisando de forma comparativa, o mapa de precipitação estimado por krigagem indicativa e o determinado pelo modelo fuzzy são significativamente distintos, com grandes variações nas escalas de probabilidade e pertinência, respectivamente. O mapa estimado por KI apresenta elevada amplitude de probabilidade, variando desde áreas onde este valor é próximo de zero até áreas onde este é próximo de um. Já no mapa estimado pelo modelo fuzzy, observa-se uma menor amplitude de pertinência, sendo que a maior parte do estado apresenta valores intermediários em relação à classe tida como ideal, que é o intervalo de 800 a $1200 \mathrm{~mm}$.

Essa variação entre os dois métodos ocorre, no caso da precipitação, por que a KI só é mensurada se o valor estiver acima ou abaixo do valor de corte $(1000 \mathrm{~mm})$ adotado no trabalho, desconsiderando 
a diferença entre regiões com precipitação acima do ideal, enquanto que para a lógica fuzzy é mensurada de forma mais detalhada, sendo seu resultado mais confiável, já que só considera ideal os valores que estão dentro do intervalo de 800 a $1200 \mathrm{~mm}$, sendo o restante integrado de forma a evidenciar quanto o valor pertence ao conjunto dos ideais. Nepomuceno (2001) afirma que o excesso de chuva pode ser, em determinadas situações, mais prejudicial às culturas agrícolas do que a própria escassez desta. Esse autor ainda relata que a análise de dados pluviométricos é um fator preponderante na definição de áreas para cultivos.

No caso da erosividade das chuvas, quase todo o estado apresenta, pelo método da krigagem indicativa, probabilidade inferior a $30 \%$ de ocorrência do valor de erosividade maiores ou iguais ao limite estabelecido neste trabalho (500 MJ.mm.ha $\left.{ }^{-1} \cdot \mathrm{h}^{-1} \cdot \mathrm{ano}^{-1}\right)$. Em linhas gerais, a região do litoral do estado é a que apresenta as maiores probabilidades de valores elevados para essa variável, sendo em alguns locais essa probabilidade igual à unidade.

Já no mapa classificado pelo sistema fuzzy, nenhuma região apresenta grau de pertinência inferior a $30 \%$, em relação ao limite de 500 MJ.mm.ha ${ }^{-1} \cdot \mathrm{h}^{-1} \cdot \mathrm{ano}^{-1}$. A quase totalidade da área apresenta pertinências próximas à unidade, diferente do observado no mapa da krigagem indicativa. Esse resultado, no caso da erosividade, se deve, exclusivamente, ao fato da lógica fuzzy trabalhar com modelos imprecisos e que buscam simular o raciocínio humano (SILVA et al., 2010a), fugindo de limites rígidos e dicotômicos. Apesar da krigagem indicativa apresentar valores de probabilidade e não valores inteiros, sua codificação inicial assemelha-se à lógica binária, onde a categorização das variáveis é realizada no sistema 0 ou 1 , sendo que, somente após a definição da dependência espacial, os dados reais assumem valores intermediários.

Independente do método utilizado, é possível observar que o estado da Bahia apresenta chuvas com grande potencial para desencadear processos erosivos, sendo necessária a adoção de diferentes técnicas de manejo e conservação do solo para não permitir que erosões ocorram. SILVA et al. (2007) comentam que o conhecimento do potencial erosivo das chuvas é o primeiro passo para o zoneamento agrícola e também para a adoção de técnicas de manejo que visam a sustentabilidade ambiental dos sistemas de produção. De acordo com esses autores, o estudo da distribuição espacial da erosividade fornece um indicativo de áreas mais susceptíveis à erosão, o que permite uma maior atenção a essas regiões, utilizando práticas que visem reduzir o risco de perda de solo nas mesmas.

\section{CONCLUSÕES}

- A metodologia utilizada para o estudo da precipitação pluviométrica e do seu potencial erosivo no estado da Bahia permitiu a obtenção de resultados satisfatórios, elevando a compreensão do fenômeno.

- A lógica fuzzy, pelo algoritmo utilizado, apresentou bom desempenho para caracterizar e mapear as incertezas tanto da precipitação pluviométrica quanto da erosividade para o estado da Bahia.

- Os valores encontrados de erosividade para o estado da Bahia foram maiores do que o limite desejável, indicando significativo potencial das chuvas em causar erosão.

\section{REFERÊNCIAS BIBLIOGRÁFICAS}

BERTONI, J. LOMBARDI NETO, F. Conservação do solo. São Paulo: Ícone Editora Ltda, 1999. 355p. BÖNISCH, S.; ASSAD, M.L.L.; CÂMARA, G.; MONTEIRO, A.M.V. Representação e propagação de incertezas em dados de solos, 1- Atributos categóricos. Revista Brasileira de Ciências do Solo, v.28, n.1, p.21-32, 2004.

BRAGA, C.C.; MELO, M.L.D.; MELO, E.C.S. Análise de agrupamento aplicada a distribuição da precipitação no estado da Bahia. In: Congresso Brasileiro De Meteorologia, 10, Brasília-DF. Anais, Sociedade Brasileira de Meteorologia. p.1857-62, 1998.

BRITTO, F.P.; BARLETTA, R.; MENDONÇA, M. Variabilidade espacial e temporal da precipitação pluvial no Rio Grande do Sul: influência do 
fenômeno el niño oscilação sul. Revista Brasileira de Climatologia, v.3, p.39-48, 2008.

CARVALHO, J.R.P.;ASSAD, E.D. Análise espacial da precipitação pluviométrica no estado de São Paulo: Comparação de métodos de interpolação. Engenharia Agrícola, v.25, n.2, p.377-384, 2005.

CHIANG, J.; HSU, J. Fuzzy classification trees for data analysis. Fuzzy Sets System, v.130, n.1, p.8799, 2002.

COSTA, H.C.; MARCUZZO, F.F.N.; FERREIRA, O.M.; ANDRADE, L.R. Espacialização e sazonalidade da precipitação pluviométrica do estado de Goiás e Distrito Federal. Revista Brasileira de Geografia Física, v.5, n.1, p.87-100, 2012.

GRAMACHO, I.C.P.; MAGNO, A.E.S.; MANDARINO, E.P.; MATOS, A. Cultivo e beneficiamento do cacau na Bahia. Ilhéus: CEPLAC, 1992. 124p.

KERRY, R.; OLIVER, M.A. Determining nugget: sill ratios of standardized variograms from aerial photographs to krige sparse soil data. Precision Agriculture, v.9, n.1, p.33-56, 2008.

LIMA, J.S.S.; SILVA, S.A.; BERNARDES, P.M.; FONSECA, A.S.; PEREIRA, J.M.S. Variabilidade espacial dos percentis 75 da precipitação pluvial mensal no estado do Espírito Santo. Engenharia na Agricultura, v.24, n.5, p.393-405, 2016.

LIMA, J.S.S.; SOUZA, G.S.; SILVA, A.S. Amostragem e variabilidade de atributos químicos do solo em área de vegetação natural em regeneração. Revista Árvore, v.34, n.1, p.127-136, 2010.

MARCUZZO, F.F.N.; ANDRADE, L.R.; MELO, D.C.R. Métodos de interpolação matemática no mapeamento de chuvas do estado do Mato Grosso. Revista Brasileira de Geografia Física, v.4, n.4, p.793-804, 2011a.

MARCUZZO, F.F.N.; CARDOSO, M.R.D.; FARIA, T.G. Chuvas na amazônia mato-grossense: análise histórica e tendência futura. Caminhos de Geografia. v.12, n.38, p.65-75, 2011 b.

MARCUZZO, F.F.N.; MELO, D.C.R.; COSTA, H.C. Sazonalidade e distribuição espaço-temporal das chuvas no bioma do cerrado do estado do Mato Grosso do Sul. Revista Brasileira de Recursos Hídricos. v.17, n.1, p.77-86, 2012.

MELLO, C.R.; VIOLA, M.R.; CURI, N.; SILVA, A.M. Distribuição espacial da precipitação e da erosividade da chuva mensal e anual no estado do Espírito Santo. Revista Brasileira de Ciência do Solo, v.36, p.1878-1891, 2012.

MENKE, A.B.; CARVALHO JUNIOR, O.B.; GOMES, R.A.T.; MARTINS, E.S.; OLIVEIRA, S.N. Análise das mudanças do uso agrícola da terra a partir de dados de sensoriamento remoto multitemporal no município de Luis Eduardo Magalhães (BA - Brasil). Sociedade \& Natureza, v.21, n.6, p.315-326, 2009.

MOLION, L.C.B.; BERNARDO, S.O. Dinâmica das chuvas no Nordeste Brasileiro. In: Congresso Brasileiro de Meteorologia, Anais... (CD-ROM). Rio de Janeiro. p. 1334-1342, 2000. Disponível em: <http://www.cbmet.com/cbm-files/12-7ea5f6 27d14a9f9a88cc694cf707236f.pdf $>$. Acesso em: 20 jul. 2017.

MOURA, M.S.B.; GALVINCIO, J.D.; BRITO, L.T.L.; SOUZA, L.S.B.; SÁ, I.I.S.; SILVA, T.G.F. Clima e água de chuva no semiárido. Petrolina: Embrapa, 2010.

NADERIANFAR, M.; PIRI, J.; KISI, O. Preprocessing data to predict groundwater levels using the fuzzy standardized evapotranspiration and precipitation index (SEPI). Water Resources Management, v.31, n.14, p.4433-4448, 2017.

NEPOMUCENO, A.L. Caracterização de risco climático nas regiões produtoras de soja no Brasil. Revista Brasileira de Agrometeorologia, v.9, n.3, p.415-421, 2001.

OLIVEIRA, N.L.; MARCUZZO, F.F.N. Influência do el niño e la niña no número de dias de precipitação 
pluviométrica do estado do Mato Grosso do Sul. Ateliê Geográfico, v.10, n.1, p.73-94, 2016.

SOUZA, W.; AZEVEDO, P. Índices de detecção de mudanças climáticas derivados da precipitação pluviométrica e das temperaturas em Recife-PE. Revista Brasileira de Geografia Física, v.5, n.1, p.143-159, 2012.

PETKOVIĆ, D.; GOCIC, M.; TRAJKOVIC, S.; MILOVANČEVIĆ, M.; ŠEVIĆ, D. Precipitation concentration index management by adaptive neuro-fuzzy methodology. Climatic Change, v.141, n.4, p.655-669, 2017.

PINTO, E.J.A.; AZAMBUJA, A.M.S.; FARIAS, J.A.M.; SALGUEIRO, J.P.B.; PICKBRENNER, K. (Coords.). Atlas pluviométrico do Brasil: isoietas mensais, isoietas trimestrais, isoietas anuais, meses mais secos, meses mais chuvosos, trimestres mais secos, trimestres mais chuvosos. Brasília: CPRM, 2011. 1 DVD. Escala 1.5:000.000. Equipe Executora: Costa, Margarida Regueira da; Dantas, Carlos Eduardo de Oliveira; Azambuja, Andressa Macêdo Silva de; Nascimento, Jean Ricardo da Silva do; Santos, André Luis M. Real dos; Farias, José Alexandre Moreira; Machado, Érica Cristina; Marcuzzo, Francisco Fernando Noronha; Medeiros, Vanesca Sartorelli; Melo, Denise Christina de Rezende; Rodrigues, Paulo de Tarso R.; Weschenfelder, Adriana Burin; Sistema de Informação Geográfica-SIG - versão 2.0 - atualizada em novembro/2011; Programa Geologia do Brasil; Levantamento da Geodiversidade. Disponível em: $<$ http://www.cprm.gov.br/publique/cgi/cgilua.exe/ sys $/$ start.htm?infoid $=1351 \&$ sid $=9>$. Acesso em: 31 jan. 2018.

RUFINO, R.L. Avaliação do potencial erosivo da chuva para o estado do Paraná: Segunda aproximação. Revista Brasileira de Ciência do Solo, v.10, p.279-281, 1986.

SILVA, A.M. Rainfall erosivity map for Brazil. Catena, v.57, p.251-259, 2004.

SILVA, S.A.; LIMA, J.S.S. Número de postos pluviométricos necessários para a estimativa da precipitação mensal no estado do Espírito Santo, Brasil. Revista Brasileira de Meteorologia, v.26, n. 4, p.555-560, 2011.

SILVA, S.A.; LIMA, J.S.S.; OLIVEIRA, R.B.; SOUZA, G.S.; SILVA, M.A. Análise espacial da erosão hídrica em um Latossolo Vermelho Amarelo sob cultivo de café conilon. Revista Ciência Agronômica, v.38, n.4, p.335-342, 2007.

SILVA, S.A.; LIMA, J.S.S.; SOUZA, G.S.; OLIVEIRA, R.B. Variabilidade espacial do potencial erosivo das chuvas para o estado do Espírito Santo, Brasil. Irriga, v.15, n.3, p.312-323, 2010a.

SILVA, S.A.; LIMA, J.S.S.; SOUZA, G.S.; OLIVEIRA, R.B.; XAVIER, A.C. Lógica fuzzy na avaliação da fertilidade do solo e produtividade do café conilon. Revista Ciência Agronômica, v.41, n.1, p.9-17, 2010 b.

SILVA, S.A.; LIMA, J.S.S.; SOUZA, G.S.; XAVIER, A.C. Distribuição e incerteza da acidez de um Latossolo Vermelho-Amarelo húmico sob cultivo de café. Revista Brasileira de Ciência do Solo, v.33, n.4, p.1053-1060, 2009.

SILVA, S.A.; LIMA, J.S.S.; ZUCOLOTO, M. Distribuição espacial das frações granulométricas em um Latossolo Vermelho Amarelo utilizando krigagem indicativa. Engenharia na agricultura, v.19, n.3, p.195-202, 2011.

VIEIRA, S.R. Geoestatística em estudos de variabilidade espacial do solo. In: Novais RF, Alvarez VH \& Schaefer CEGR. Tópicos em ciência do solo, Viçosa - MG: Sociedade Brasileira de Ciência do Solo, 1:1-54, 2000.

ZIMBACK, C.R.L. Análise espacial de atributos químicos de solos para fins de mapeamento da fertilidade do solo. Tese de Doutorado. Faculdade de Ciências Agronômicas, Universidade Estadual Paulista. Botucatu. 114p, 2001. 